\title{
Growth Optimization, Strain Compensation and Structure Design of InAs/GaSb Type-II Superlattices for Mid-Infrared Imaging
}

\author{
Yuxin Song ${ }^{1 *}$, Shumin Wang ${ }^{1,2^{*}}$, Carl Asplund ${ }^{3}$, Rickard Marcks von Würtemberg ${ }^{3}$, \\ Hedda Malm ${ }^{3}$, Amir Karim ${ }^{4}$, Xiang $\mathrm{Lu}^{5}$, Jun Shao ${ }^{5}$ \\ ${ }^{1}$ Department of Microtechnology and Nanoscience, Photonics Laboratory, \\ Chalmers University of Technology, Gothenburg, Sweden \\ ${ }^{2}$ State Key Laboratory of Functional Materials for Informatics, Shanghai Institute of \\ Microsystem and Information Technology, Chinese Academy of Sciences, Shanghai, China \\ ${ }^{3}$ IRnova AB, Kista, Sweden \\ ${ }^{4}$ Acreo AB, Kista, Sweden \\ ${ }^{5}$ National Laboratory for Infrared Physics, Shanghai Institute of Technical Physics, CAS, Shanghai, China \\ Email: "yuxin.song@chalmers.se, shumin@chalmers.se
}

Received February 15, 2013; revised March 20, 2013; accepted March 29, 2013

Copyright (C) 2013 Yuxin Song et al. This is an open access article distributed under the Creative Commons Attribution License, which permits unrestricted use, distribution, and reproduction in any medium, provided the original work is properly cited.

\begin{abstract}
InAs/GaSb type-II superlattce (T2SL) photodetector structures at the MWIR regime were grown by molecular beam epitaxy. The growth temperature and group- $\mathrm{V}$ soaking times were optimized with respect to interface and transport quality. Novel strain compensation schemes with insertion of InSb layers were proposed and tested to be efficient to tune the overall strain between tensile and compressive without degradation of interface and optical quality. The effect of the proposed methods is modeled by analytic functions. Band structure calculations were also carried out for the proposed T2SL structures to assist optimizing sample designs. Single pixel photodiodes with a low dark current were demonstrated.
\end{abstract}

Keywords: InAs/GaSb; Type-II Superlattce; Molecular Beam Epitaxy; Strain Compensation

\section{Introduction}

In the two atmospheric transmission windows at a wavelength range of $3-5$ and $8-14 \mu \mathrm{m}[1]$, there is a large demand for imaging within industrial, biomedical, civilian-surveillance and military applications. The most developed materials are $\mathrm{Cd}_{\mathrm{x}} \mathrm{Hg}_{1-\mathrm{x}} \mathrm{Te}(\mathrm{MCT})$, InSb and quantum well infrared photodetectors (QWIPs) [2-4]. Type-II superlattices (T2SL) comprising combinations of (Al, Ga, $\mathrm{In}) \mathrm{Sb}$ and InAs layers have received increased interest the last decade as a promising candidate in photodetector applications. InAs/GaSb T2SL structure was first proposed by Sai-Halasz et al. in 1977 [5] and suggested to be a candidate for infrared detection with potentials for superior performance and production yield compared with MCT by Smilth and Mailhiot in 1987 [6]. This is especially true for applications in the long-wave infrared (LWIR) atmospheric transmission window of $8-12 \mu \mathrm{m}$,

${ }^{*}$ Corresponding authors. where the difficulty of maintaining sufficient composition control of MCT leads to low production yields, and where the low quantum efficiency of quan- tum-well infrared (QWIP) detectors preclude their use for certain critical applications. For imaging in the mid- wave infrared (MWIR) transmission window of $3-5 \mu \mathrm{m}$, on the other hand, there are already two well established, competing technologies with rather high manufacturability: large-area, high quality focal plane detectors of InSb are available, but they require cooling typically to $80 \mathrm{~K}$. $\mathrm{MCT}$ detectors operate with similar signal-to-noise ratios at temperatures beyond $100 \mathrm{~K}$, which means longer cooler lifetime and reduced power consumption. However, the use of the new material T2SL can be motivated also for this wavelength range, since it already surpasses performance of InSb by a wide margin, and is compatible with standard III-V processes and processing tools, unlike MCT. Furthermore, T2SL holds-at least theoretically-promise of even higher operating temperatures than 
MCT from strongly reduced Auger recombination. The provision is that the density of the as-yet unidentified Shockley-Read-Hall centers can be decreased significantly.

InAs and $\mathrm{GaSb}$ are well known III-V materials with established processing technologies. The closely matched lattice constants make T2SL materials possible to be grown by epitaxy without introducing large extra strain. By changing the layer thicknesses, the effective band gap can be tuned from $0.5 \mathrm{eV}$ to $0 \mathrm{eV}$ (semi-metallic) [7]. Detectors with a cutoff wavelength as long as $32 \mu \mathrm{m}$ have been demonstrated [8]. The close lattice constants also allow for integrating multiple structures with different wavelengths in the same device, resulting in multicolor detectors and cameras [9]. Tunneling currents in T2SLs can be reduced due to a large electron effective mass. Moreover, Auger recombination can be suppressed to a large extent owing to the large valence band splitting [10]. T2SL structures can also provide high quantum efficiency. The responsivity of T2SL detectors has already proved to be similar to those made of MCT [11]. Thanks to modern molecular beam epitaxy (MBE) technology, T2SL materials can be grown with a very low defect density and high uniformity, making it especially suitable for fabrication of FPAs [12]. During the period 1996 to 2005, significant developments in growth and fabrication of T2SL structures were achieved, leading to the first demonstration of a T2SL based FPA in 2005 [13]. Since then, interest has grown significantly in development of T2SL based detectors and FPAs [9,14-17].

One major challenge for successful growth is the control of overall strain, primarily caused by the small lattice mismatch between InAs and $\mathrm{GaSb}[18,19]$ and the chemical nature at $\mathrm{InAs} / \mathrm{GaSb}$ interfaces. Strain relaxation and formation of dislocations can occur if the overall strain is not effectively compensated in a several micrometers thick detector structure. The InAs/GaSb interface can be of GaAs type (tensile strain) or InSb type (compressive strain). Some strain compensation methods were tested. The main idea is to increase the percentage of InSb at interfaces to introduce compressive strain, thus compensating the accumulated tensile strain caused by InAs and by GaAs-type interfaces. Plis et al. studied the strain compensation by using $\mathrm{Sb}$ soaking after the growth of InAs layer to covert part of the InAs into InSb [20]. The method of intentionally inserting a thin InSb layer after the growth of InAs layer was utilized by Rodriguez [21], Haugan [22], Plis [23] et al. Interface quality also plays a very important role for T2SL-based device performance, and therefore large efforts were made toward understanding and improving the quality of interfaces [18] [24-26]. The effects of soaking using group- $\mathrm{V}$ elements at interfaces on interface quality and overall strain were studied $[20,27]$. Moreover, control of background doping level [28-30] and influence of substrate quality on device performance were also investigated [31,32].

In this paper, we present optimization of growth parameters and strain compensation of InAs/GaSb T2SLs grown by MBE aiming for fabricating mid-wavelength infrared (MWIR) FPA devices. The MBE system was designed for growth of both As- and Sb-compounds and a heavy background As pressure is expected. This will make it more difficult for effective strain compensation compared with a dedicated MBE system with a minimum effect of background As. Novel strain compensation schemes with insertion of one monolayer InSb layer inside the GaSb layer, at the $\mathrm{InAs} / \mathrm{GaSb}$ and $\mathrm{GaSb} / \mathrm{InAs}$ interfaces and their combinations are proposed, modeled, analyzed and tested, and their influence on structural and optical qualities is compared. We find that proper combinations of the proposed schemes can control interface quality of InAs/GaSb T2SLs and tune the overall strain between tensile and compressive. Band structure calculations for the proposed structures were carried out to assist understanding and future improvements. Finally, single pixel photodetectors with a low dark current were demonstrated using the optimized growth conditions.

\section{Experiments}

\subsection{Sample Growth}

All the samples were grown by a Riber Compact21 MBE system with a cluster tool, equipped with dual filament effusion cells for In and Ga and valved crackers for As and $\mathrm{Sb}$. To lower the substrate cost at the initial optimizations of growth parameters, semi-insulation $\mathrm{GaAs}(100)$ substrates were employed. The GaAs substrates were first de-oxidized at $660^{\circ} \mathrm{C}$ (all growth temperatures used in this paper were read by a thermocouple) followed by a $100 \mathrm{~nm} \mathrm{GaAs}$ buffer layer grown at $620^{\circ} \mathrm{C}$ to smoothen the growth front. The substrate temperature was then ramped down to $510^{\circ} \mathrm{C}$ with the As valve closed. An atomic monolayer of $\mathrm{Ga}$ was deposited to achieve groupIII-rich surface reconstruction and then a $1 \mu \mathrm{m} \mathrm{GaSb}$ buffer layer was grown using the interfacial misfit array (IMF) method [33] to accommodate the $7.8 \%$ lattice mismatch between GaAs and GaSb and to obtain smooth surface with a low defect density. Latter samples were grown on n-type GaSb (100) substrates. The GaSb substrates were de-oxidized at $580^{\circ} \mathrm{C}$ followed by a $100 \mathrm{~nm}$ $\mathrm{GaSb}$ buffer layer grown at $510^{\circ} \mathrm{C}$. The T2SL structures were grown on the buffer layer at varied, but much lower growth temperatures $\left(\mathrm{T}_{\mathrm{g}}\right)$. Reflection high-energy electron diffraction (RHEED) was used for in-situ monitoring of the growth process.

\subsection{Structural Property Characteriztion}

X-ray diffraction (XRD) based on PANalytical X'Pert 
PRO X-ray diffraction system was employed as the main tool to characterize the structural properties. As most physical processes occur at, or close to, the interfaces between InAs and GaSb layers in the superlattice, the interface quality is one of the major parameters for structural quality and can be quantified by measuring the full width at half maximum (FWHM) of the diffraction fringes from the superlattice in XRD rocking curves. The narrower the diffraction peaks, the smoother the interfaces. Moreover, the separation between the GaSb substrate (or GaSb buffer layer) peak and the 0th order diffraction peak of the superlattice in XRD rocking curves can be used to measure the overall lattice mismatch of a T2SL to GaSb. Although the lattice constants of InAs and GaSb are very close (6.0960 vs $6.0583 \AA$ ), there is still $0.6 \%$ lattice mismatch (tensile strain) when growing InAs on GaSb. Different types of interfaces discussed in detail below may also bring in additional strain. Strain relaxation may occur for micrometer thick devices. An example of this can be seen in the XRD rocking curves of a sample grown on GaAs in Figure 1(a), where each superlattice peak is split into two. A cross-sectional

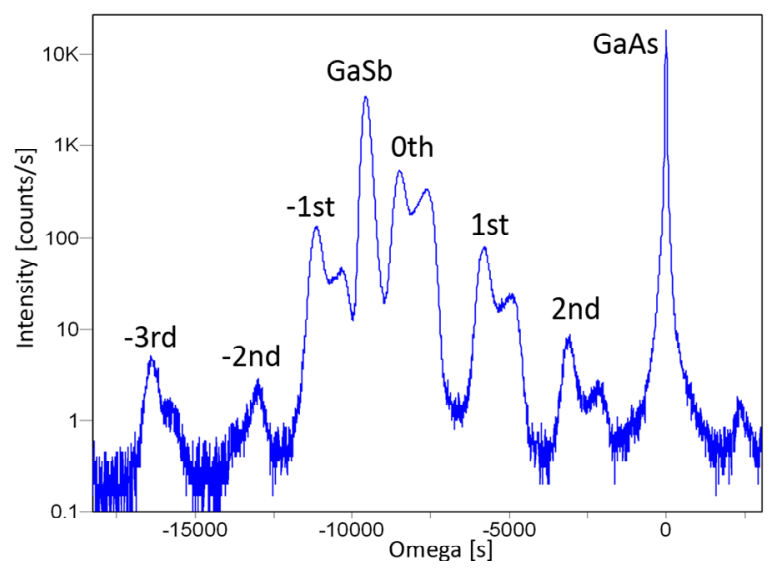

(a)

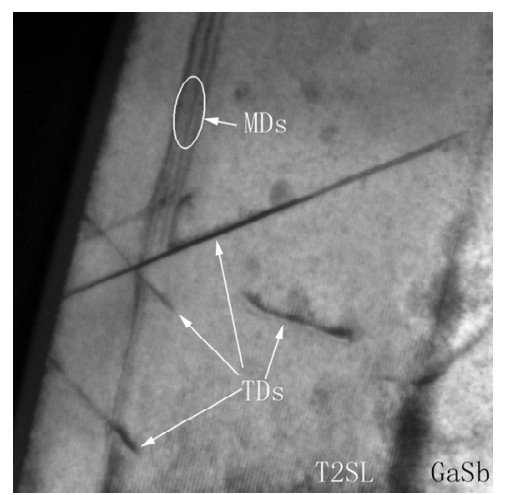

(b)

Figure 1. (a) XRD rocking curve of one partially relaxed T2SL sample (100 periods of $10 \mathrm{ML} \mathrm{GaSb/10} \mathrm{ML} \mathrm{InAs)}$ grown on a GaAs substrate, and (b) an XTEM image of the same sample. Both MDs and TDs are marked. transmission electron microscopy (XTEM) image shown in Figure 1(b) confirms that the upper part of the T2SL structure is partially relaxed by nucleation of misfit dislocations (MDs). These MDs are usually accompanied by threading dislocations (TDs) terminated on the sample surface. The two sets of diffraction peaks correspond to $\mathrm{X}$-ray interference from the upper relaxed and the lower strained superlattices, respectively. Simulation and fitting of the XRD rocking curves for T2SLs is possible, but trivial. As the total thickness of one period of a T2SL structure for MWIR detection is commonly less than 20 $\mathrm{ML}$, the two interfaces compose a significant portion of strain contribution in each period and their chemical compositions are usually unknown in simulation and have to be regarded as fitting parameters. We found that the calibration of InAs and GaSb growth rates based on the periodicity of the superlattice diffraction peaks is no longer accurate. RHEED oscillation is recommended for calibration of the GaSb and InAs growth rate.

\subsection{Optical Property Characterization}

Photoluminescence (PL) at $77 \mathrm{~K}$ was used to examine the bandgap as well as the optical quality of the T2SL material. The PL measurements were based on a Bruker/Fourier transform infrared spectroscopy (FTIR) Equinox 55 system with Hyperion microscope.

\section{Optimization of Growth Conditions}

Growth temperature is one of the most important parameters for MBE growth of T2SL structures. Figure 2(a) compares XRD rocking curves of the two samples grown at $340^{\circ} \mathrm{C}$ and $470^{\circ} \mathrm{C}$, respectively. It can be found that the one grown at the low temperature shows better structural quality with narrow diffraction peaks and clear high order peaks, while the one grown at the high $\mathrm{T}_{\mathrm{g}}$ has an inferior superlattice structure judged by broadening of the diffraction peaks. High growth temperature will enhance inter-diffusion between different elements and also segregation of large atoms i.e. In and $\mathrm{Sb}$, leading to deteriorated interface quality. Therefore it is essential to employ low growth temperatures to obtain sharp interfaces. Due to different heater designs and temperature measurement techniques employed in different MBE systems, the nominal $\mathrm{T}_{\mathrm{g}}$-values reported in the literature are unreliable. We used the temperature and $\mathrm{Sb}$ flux dependent $(1 \times 3)$ to $(2 \times 5)$ surface reconstruction transition on $\mathrm{GaSb}$, described by Bracker et al. [34], as the temperature reference. The proper growth temperature is commonly lower than the transition temperature under a certain $\mathrm{Sb}$ flux [10]. We optimized the growth temperature in the range of $320^{\circ} \mathrm{C}-380^{\circ} \mathrm{C}$ and the XRD, atomic force microscopy (AFM) and Hall measurement results are summarized in Figures 2(b) and (c), respectively. It can be found from 


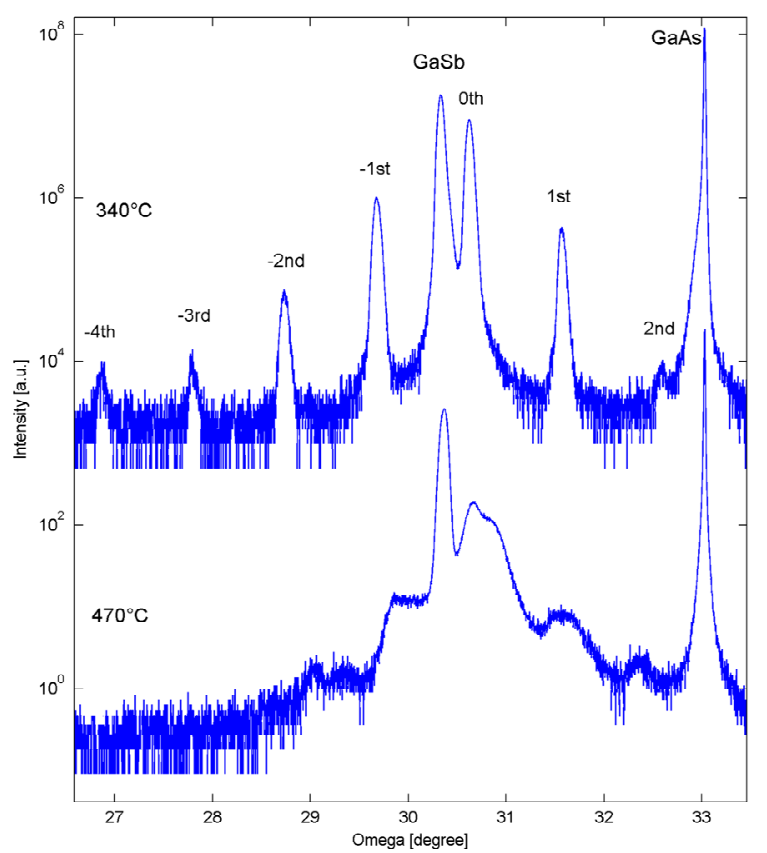

(a)

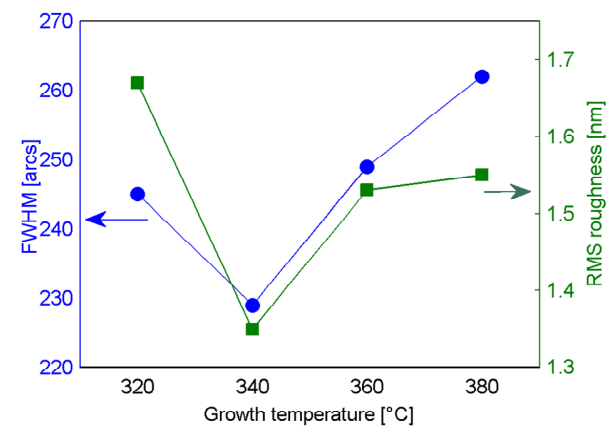

(b)

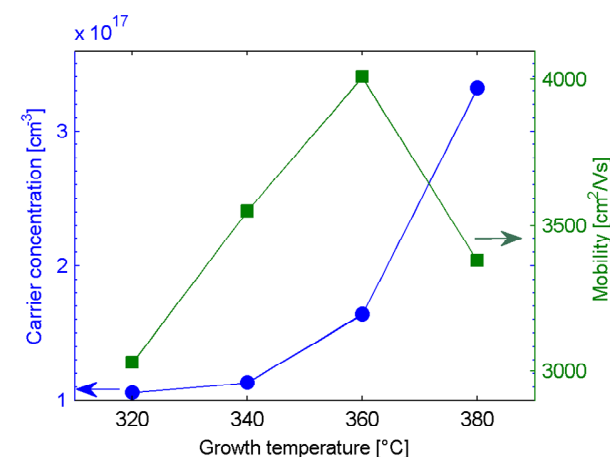

(c)

Figure 2. Effects of growth temperature on interface and transport quality of T2SL structures on GaAs comprising 100 periods of $10 \mathrm{ML}$ InAs/10 ML GaSb. (a) shows XRD(004) rocking curves grown at $340^{\circ} \mathrm{C}$ and $470^{\circ} \mathrm{C}$, respectively. (b) shows the FWHM of the $-1^{\text {st }}$ order diffraction peaks (blue round) and the RMS roughness values measured in $10 \times 10 \mu \mathrm{m}^{2}$ AFM scans (green square) vs. $T_{g}$. (c) shows the carrier density (blue round) and mobility (green square) vs. $T_{g}$. All measurements were carried out at room temperature.
Figure 2(b) that the sample grown at $340^{\circ} \mathrm{C}$ has both the best interface quality as indicated by the narrowest -1 st order diffraction peak and the smoothest surface. Growth temperature also has an effect on carrier density and mobility, as shown in Figure 2(c). The general trend is that the carrier density increases with $\mathrm{T}_{\mathrm{g}}$, while the electron mobility reaches a maximum at $360^{\circ} \mathrm{C}$. We chose $\mathrm{T}_{\mathrm{g}}=$ $340^{\circ} \mathrm{C}$ for the later samples. This temperature is much lower than the optimal temperature for growing $\mathrm{GaSb}$.

The residual doping of an InAs bulk layer is normally $n$-type, while it is $p$-type for a GaSb bulk layer, presumably due to the Fermi level pinning. The residual carrier density in an InAs/GaSb T2SL is determined by the compensation of unintentionally doped carriers in $\mathrm{GaSb}$ and InAs layers [29]. The optimal $\mathrm{T}_{\mathrm{g}}$-value for InAs/ $\mathrm{GaSb}$ T2SLs is far below the optimal $\mathrm{T}_{\mathrm{g}}$-values for $\mathrm{GaSb}$ and InAs bulk materials, leading to possibly a higher density of impurities in both layers than those grown at elevated growth temperatures. The measured net $n$-type carriers indicate that there are more electrons contributed by InAs than the holes by GaSb. It's still unclear why the carrier density increases with growth temperature while the mobility has a maximum at $360^{\circ} \mathrm{C}$.

Due to the fact that dual group- $\mathrm{V}$ elements are involved in growth of T2SL materials, soaking of one element at the interface is commonly used and necessary for tuning the overall strain. Arsenic soaking after growth of $\mathrm{GaSb}$ will strengthen GaAs-like interface leading to extra tensile strain in the structure. However, it has been reported that the As soaking can help in obtaining high crystalline and optical quality [27]. We have tested four different As soaking times with otherwise the same growth conditions. The $\mathrm{XRD}(004)$ rocking curves shown in Figure 3 reveal huge differences between samples with and without As soaking. For the sample grown without As soaking, the interfaces have very low quality with

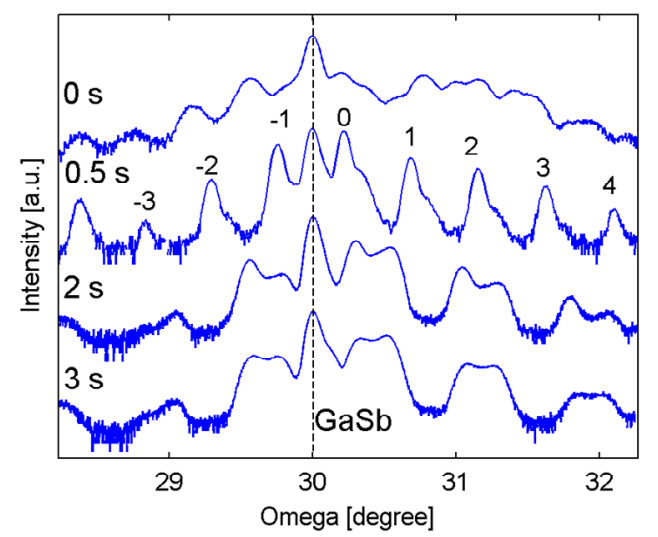

Figure 3. XRD (004) rocking curves of T2SLs composed of 100 periods $10 \mathrm{ML}$ InAs/10ML GaSb grown at $340^{\circ} \mathrm{C}$ on GaAs substrates with different As soaking time labeled above each curve. The order of the diffraction peaks for the sample with $0.5 \mathrm{~s}$ As soaking is marked above the curve. 
almost no clear diffraction peaks. However, as short as 0.5 second As soaking makes a significant difference.

This sample has well shaped high order diffraction peaks. Further increasing the As soaking time to $2 \mathrm{~s}$ and $3 \mathrm{~s}$ leads to peak splitting, indicating strain relaxation possibly caused by As-soaking induced excess tensile strain. This underlines the importance of strain compensation, which will be discussed in detail in the next section. For most of the following samples, 0.5 second As soaking time was employed after growth of $\mathrm{GaSb}$. It should be noted that the proper As soaking time is related to the As flux used as well as the background As during the growth of GaSb. For the optimization shown in Figure 3, an Addon As-cracker was used. This cracker has an inefficient cracking efficiency and a severe leakage when the needle valve was closed leading to a high As background. When a Riber As-cracker was used later, the background As was reduced significantly and the optimal As-soaking may shift away from 0.5 second.

Antimony soaking after growth of the InAs layer up to 14 seconds was also tested. No obvious difference was observed. This is probably due to the large amount of accumulated As on the InAs surface, such that the Sbsoaking becomes ineffective. 12-second Sb soaking time was employed for most of the later samples.

\section{Strain Compensation}

Although InAs and $\mathrm{GaSb}$ are commonly regarded as a "lattice matched" material system, the $0.6 \%$ lattice mismatch still leads to overall tensile strain in the structure when grown on GaSb substrates and strain relaxation may occur when it is accumulated in micrometer thick devices. Threading dislocations will then strongly degrade the device performance. Therefore, a strain compensation scheme to avoid formation of TDs is essential for T2SL structures.

Different types of interfaces can also bring in excess strain. These, however, can be engineered. There are basically two types of interfaces in InAs/GaSb T2SL structures, GaAs-like and InSb-like. Due to the different bond lengths, GaAs-like interfaces will add tensile strain, while InSb-like interfaces bring in compressive strain. Apparently, an InSb-like interface is preferable to compensate the accumulated tensile strain of InAs. Use of group-V-rich flux is the common condition for $\mathrm{MBE}$ growth of III-V compounds, i.e. each layer of InAs and $\mathrm{GaSb}$ is group $\mathrm{V}$ terminated. When growth of an InAs layer is finished, the top atomic layer is As. The next incorporated atomic layer will be the group-III element, $\mathrm{Ga}$, when the growth of a GaSb layer is initiated. Consequently, the InAs/GaSb interface is connected by As-Ga bonds, forming a GaAs-like interface as shown in Figure 4(a). One would expect an InSb-like interface to form in a similar way when an InAs layer is grown on a $\mathrm{GaSb}$ layer, as shown in Figure 4(b). However, since the substitution of $\mathrm{Sb}$ atoms by As atoms is thermodynamically favored, the result can be a partial or complete GaAs-like interface also in this case; see Figure 4(c). Therefore, the chance to form GaAs-like interfaces is usually higher than that for InSb-like interfaces, leading to increased overall tensile strain, i.e. the 0th peak appears at a larger angle than that of the GaSb peak.

For a small lattice mismatched heterostructure grown at low growth temperatures, it is reasonable to assume that there is little intermixing between group-III atoms through diffusion or segregation. For an ordinary InAs/ GaSb T2SL structure shown in Figure 5(a), the number of In and $\mathrm{Ga}$ atomic layers in each period is denoted by $M$ and $N$, respectively. We assume that $\mathrm{As}$ and $\mathrm{Sb}$ intermixing only occurs at the interface to form one atomic layer of AsSb and let $x$ and $y$ to be the As composition at the $\mathrm{GaSb} / \mathrm{InAs}$ and the InAs/GaSb interfaces, respectively. Subsequently, for the last Ga layer at the $\mathrm{GaSb}$ / InAs interface, the bottom (left in the figure) bonds are Ga-Sb bond, while the up (right) bonds become partially Ga-As and Ga-Sb bonds with proportion of $x$ and $(1-x)$, respectively. For the first In layer, the bottom

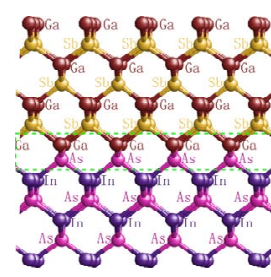

(a)

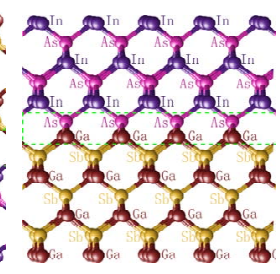

(b)

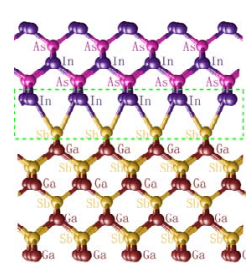

(c)
Figure 4. Schematic illustrations of different types of interfaces: (b) InSb-like interface, (a) and (c) GaAs-like interface.

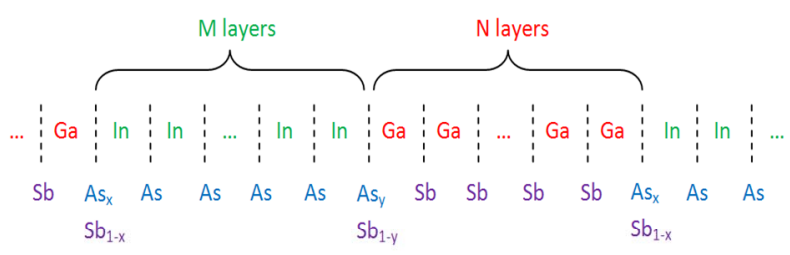

(a)

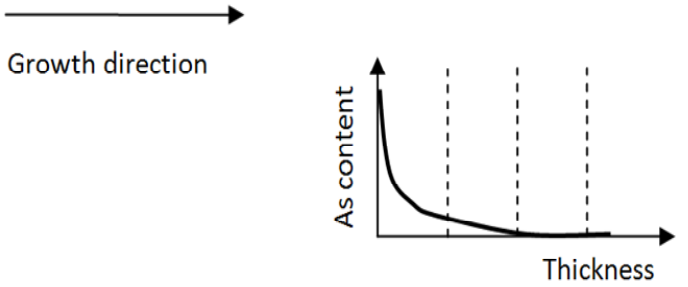

(b)

Figure 5. Schematics of (a) an InAs/GaSb T2SL structure, and (b) incorporation of residual As into the following GaSb layer. 
bonds are $x$ In-As and $(1-x)$ In-Sb while the up bonds are still In-As. It is a similar situation for the other inter lattice mismatch can be expressed as

$$
f=\left[\left(M-1+\frac{x}{2}+\frac{y}{2}\right) f_{I n A s}+\left(\frac{x}{2}+\frac{y}{2}\right) f_{G a A s}+\left(\frac{1-x}{2}+\frac{1-y}{2}\right) f_{I n S b}\right] /(M+N)
$$

where $f_{\text {InAs }}, f_{\text {GaAs }}$ and $f_{\text {InSb }}$ are the lattice mismatch of InAs, GaAs and InSb with respect to $\mathrm{GaSb}$, respectively. $(M+N)$ is the total number of atomic monolayers of one period of the T2SL structure. In the above derivation, we neglect the small difference in lattice constants of InAs and $\mathrm{GaSb}$ in the denominator. For an MBE chamber used to grow arsenides, a heavy amount of background As is expected to be incorporated during the growth of the GaSb layer, forming $\mathrm{GaSb}_{1-\mathrm{z}} \mathrm{As}_{\mathrm{z}}$, where $z$ is the composition of As. Moreover, at such low growth temperatures, which are comparable to the As bulk temperature, the adsorbed As atoms may not be capable of re-evaporating from the sample surface if not incorpo- face. The In-As, Ga-As and In-Sb bonds all contribute to the lattice mismatch to the GaSb substrate. The overall

$$
f=\left[\left(M-1+\frac{x}{2}+\frac{y}{2}\right) f_{I n A s}+\left(\frac{x}{2}+\frac{y}{2}+N z^{\prime}\right) f_{G a A s}+\left(\frac{1-x}{2}+\frac{1-y}{2}\right) f_{I n S b}\right] /(M+N)
$$

where, $z^{\prime}=\alpha / N+z$ is the average background As composition in GaSb and $\alpha$ is the total amount of incorporated As in GaSb layer due to the excess As atoms rated, leading to a certain amount of residual adsorbed As atoms on the growth front of the InAs layer. Part of these adsorbed As atoms will be incorporated into the following GaSb layer as schematically shown in Figure 5(b). A similar process could occur at the other interface for $\mathrm{Sb}$ atoms, but the effect will be much weaker due to the weak bonding of Ga-Sb compared with Ga-As and is negligible here. In addition, the background $\mathrm{Sb}$ in InAs is much lower than the background $\mathrm{As}$ in GaSb, judged by at least one order of magnitude difference in background pressure when growing GaSb and InAs. As a result, additional tensile strain will be introduced by the adsorbed and background As atoms. Then, Equation (1) becomes

$$
f=\left(0.0691-0.0062 M-0.0709 x-0.0709 y-0.0726 N z^{\prime}\right) /(M+N)
$$

Before applying strain compensation, the overall strain is measured to be tensile for $M=10$, i.e. $f<0$. Therefore, the key idea for strain compensation is to reduce the values of $x$ and $y$, as well as using a small As flux to minimize

Strengthening the InSb-like interfaces by extensive $\mathrm{Sb}$ soaking to reduce $y$-value is a common strategy for strain compensation in T2SL structures. By using long time $\mathrm{Sb}$ soaking after growth of InAs layers, part of the top As atoms would be replaced by $\mathrm{Sb}$ atoms, forming partially InSb-like interfaces. Figure 6(a) shows the shutter se- quence of this method. The overall lattice mismatch can be expressed in the same form as Equations (2) and (3). It is easily seen that the strain can never be fully compensated by only $\mathrm{Sb}$ soaking even with both $x$ - and $y$-values equal to zero if the InAs thickness is more than $11 \mathrm{ML}$. Sb-As exchange during the Sb soaking has been found to be much less effective than As-Sb exchange during the As soaking [20,27]. Therefore, we expect that the value of $y$ is high, or even close to 1 . If we assume that $y=1$, Equation (3) reduces to

$$
f=\left(-0.0018-0.0062 M-0.0709 x-0.0726 N z^{\prime}\right) /(M+N)
$$

This indicates that $f$ can never reach zero for any thickness of InAs using only soaking. We thus propose new strain compensation methods based on shutter sequences as schematically shown in Figures 6(b)-(d). We introduce an additional In layer to reinforce the InSb-like interfaces. In method (b), the $1 \mathrm{ML}$ In is deposited right after $\mathrm{GaSb}$, and then soaked with $\mathrm{Sb}$ to form $1 \mathrm{ML} \mathrm{InSb}$. The short As soak before switching to InAs leads to formation of a monolayer of InAsSb due to As-Sb intermixing. In method (c), a short $\mathrm{Sb}$ soaking is employed after the growth of InAs layer to remove residual As and establish an $\mathrm{Sb}$ environment on the growth front. Then the $1 \mathrm{ML}$ In is deposited followed by Sb soaking again. Thus, a full monolayer of $\mathrm{InSb}$ is formed. In both cases, formation of GaAs-like interfaces can be minimized. In method (d), the $1 \mathrm{ML}$ InSb is directly inserted in the middle of GaSb layer, as far as possible from the As-containing interfaces. The overall lattice mismatch in each of these methods can be expressed below in Table 1.

Since one additional monolayer of In is inserted for methods (b), (c) and (d), the total thickness in each period is changed to $(M+N+1)$. If we compare the first 
Table 1. Expressions of overall lattice mismatch in different strain compensation methods.

\begin{tabular}{|c|c|}
\hline Methods & Overall lattice mismatch \\
\hline (a) & $\begin{array}{l}f=\left[\left(M-1+\frac{x}{2}+\frac{y}{2}\right) f_{\text {InAs }}+\left(\frac{x}{2}+\frac{y}{2}+N z^{\prime}\right) f_{\text {GaAs }}+\left(\frac{1-x}{2}+\frac{1-y}{2}\right) f_{I n S b}\right] /(M+N) \\
f=\left(0.0691-0.0062 M-0.0709 x-0.0709 y-0.0726 N z^{\prime}\right) /(M+N)\end{array}$ \\
\hline (b) & $\begin{array}{l}f=\left[\left(M-1+x+\frac{y}{2}\right) f_{\text {InAs }}+\left(\frac{y}{2}+N z^{\prime}\right) f_{\text {GaAs }}+\left(2-x-\frac{y}{2}\right) f_{\text {InSb }}\right] /(M+N+1) \\
f=\left(0.132-0.0062 M-0.0691 x-0.0709 y-0.0726 N z^{\prime}\right) /(M+N+1)\end{array}$ \\
\hline (c) & $\begin{array}{l}f=\left[\left(M+\frac{X}{2}\right) f_{\text {InAs }}+\left(\frac{X}{2}+N z^{\prime}\right) f_{\text {GaAs }}+\left(1-\frac{X}{2}\right) f_{\text {InSb }}\right] /(M+N+1) \\
f=\left(0.0629-0.0062 M-0.0709 x-0.0726 N z^{\prime}\right) /(M+N+1)\end{array}$ \\
\hline (d) & $\begin{array}{l}f=\left[\left(M-1+\frac{x}{2}+\frac{y}{2}\right) f_{\text {InAs }}+\left(\frac{x}{2}+\frac{y}{2}+N z^{\prime}\right) f_{\text {GaAs }}+\left(2-\frac{x}{2}-\frac{y}{2}\right) f_{\text {InSb }}\right] /(M+N+1) \\
f=\left(0.132-0.0062 M-0.0709 x-0.0709 y-0.0726 N z^{\prime}\right) /(M+N+1)\end{array}$ \\
\hline
\end{tabular}

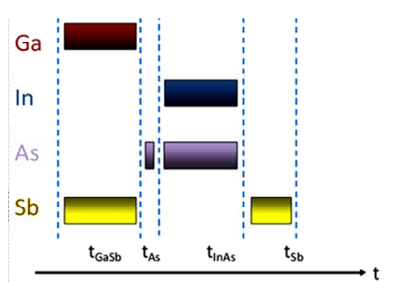

(a)

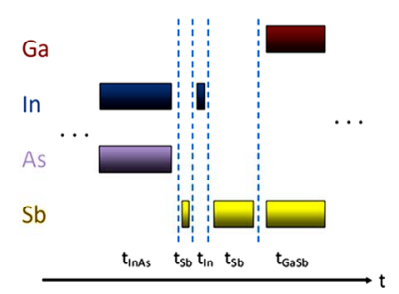

(c)

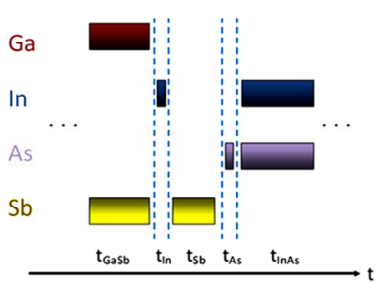

(b)

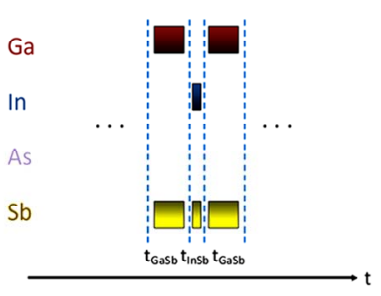

(d)
Figure 6. Shutter sequences of different strain compensation methods. (a) Common way with As and Sb soakings at interfaces only. (b) Strengthened In(As)Sb-type interface after a GaSb layer. (c) Strengthened In(As)Sb-type interface after an InAs layer. (d) One additional InSb monolayer inserted in the middle of a $\mathbf{G a S b}$ layer.

equation of each method, the first term with $f_{\text {InAs }}$ does not differ very much in all the cases. The second term with $f_{\text {GaAs }}$ is reduced to roughly half in (b) and (c). This is a direct evidence that insertion of $1 \mathrm{ML} \mathrm{InSb}$ at one interface prevents direct contact of As and Ga atoms from forming GaAs-like interface. The last term with $f_{\text {InSb }}$ reflects the effect of the one monolayer InSb.

$f, M$ and $N$ are variables, which can be controlled or measured by XRD. So they can be considered as known values. By changing the $\mathrm{GaSb}$ thickness, i.e. $\mathrm{N}$, but keeping all other growth parameters the same, the average residual As composition in the GaSb layer, $z^{\prime}$, can be obtained with any methods discussed above. If we assume that the overall lattice mismatch of an original T2SL structure is $f_{1}$, and that of another structure with the GaSb layer thickness changed by $\Delta N$, is $f_{2}$, we can calculate the $z^{\prime}$ as

$$
z^{\prime}=\frac{(M+N) f_{1}-(M+N+\Delta N) f_{2}}{0.0726 \Delta N}
$$

It is obvious from Figure 6 that, under the same growth conditions, the $x$-value should be the same for methods (a), (c) and (d), while the $y$-value should be the same for (a), (b) and (d). $z^{\prime}$ value should be the same for all the cases as long as the same As flux is used. Upon knowing the $z^{\prime}$-value, both $x$ - and $y$-values can be readily obtained by solving the equations. This provides quantitative information of chemical compositions at the two interfaces and indicates the effectiveness for the particular strain compensation technique used. The above arguments also indicate that strain compensation can be implemented in a controlled manner, making it easy to balance strain compensation with other requirements for a detector, such as bandgap, optical and structural qualities.

The strain compensation methods illustrated in Figure 6 were tested individually on the first batch of $\mathrm{GaSb}$ based samples with 100 periods of $10 \mathrm{ML}$ InAs/10 ML $\mathrm{GaSb}$ layers. When one method was applied at one interface, the other interface was treated with the normal soaking. The FWHM of the -1 st order diffraction peak for the sample with method (a) is 165 arcs, and the residual lattice mismatch is $-0.51 \%$. It turns out that the strain compensation method (b), (c) and (d) indeed reduces overall strain compared with (a) by $49 \%, 78 \%$ and $41 \%$, respectively. Method (c) shows the largest strain compensation effect, but the FWHM of the -1 st order 
diffraction peak is 2.3 times compared to that of method (a). Method (b) and (d) show narrower -1 st diffraction peaks than that of method (a) by $34 \%$ and $76 \%$, respectively, despite being less effective in strain compensation. Using above equations, we find $y=95 \%$ in method (a), close to 1 as we expect, and $x+1.025 \mathrm{Nz}^{\prime}=26 \%$ in method (c). This implies that at most $26 \% \mathrm{Sb}$ was replaced by As during the $0.5 \mathrm{~s}$ As soaking and only $5 \%$ As is replaced by $\mathrm{Sb}$ when the InAs surface is soaked by $\mathrm{Sb}$ for $12 \mathrm{~s}$. A PL comparison for the samples using the scheme (a) and (d) was shown in Figure 7. The sample grown with the method (d) has better interface quality and smaller lattice mismatch, and shows a much higher PL intensity than that with the method (a). Since the sample grown with the method (d) has one monolayer of InSb embedded in the GaSb layer, the PL peak wavelength is red-shifted compared to the sample grown with the method (a).

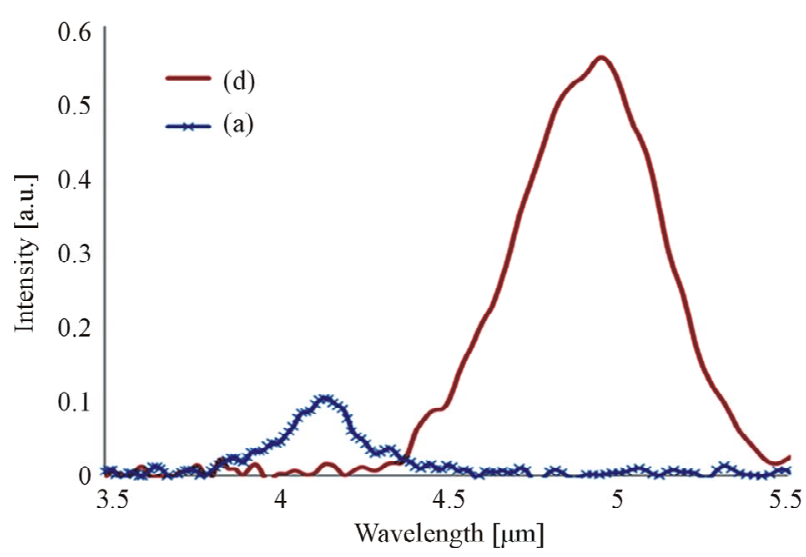

Figure 7. PL results at $77 \mathrm{~K}$ of samples with the strain compensation method (a) and (d). Both samples are grown on GaSb substrates with 100 periods of 10 ML InAs and 10 ML GaSb.
Strain compensation schemes with combinations of the above methods were investigated to further compensate the overall strain. The overall lattice mismatch can be expressed in the same way in Table 2.

The results on structural properties of this group of samples are summarized in Table 2. It should be noted that the thickness of InAs layers in this group of samples is $7 \mathrm{ML}$ in order to adjust the PL wavelength to be shorter than $5 \mu \mathrm{m}$. The interface quality is surprisingly improved, compared to the case of employing one strain compensation method (b) or (c). Both the combinations of (b) + (d) and (c) + (d) show very small residual lattice mismatch and narrow -1 st order diffraction peaks indicating high interface quality. Combination of (b) + (c) leads to slightly larger residual lattice mismatch and worse interface quality. By combining all the methods (b) $+(c)+(d)$, the strain is tuned from tensile type to compressive type with relative good interface quality. Validation of the equations was tested with these measurement data. For example, for methods (b) $+(\mathrm{c})$ and $(\mathrm{b})+(\mathrm{c})+$ (d), they should have the same interface conditions. The calculated values of $x+1.05 \mathrm{Nz}^{\prime}$ from the equations fall within a deviation of only $6 \%$. The contribution of each strain compensation method alone can be extracted through the above equations. The overall lattice mismatch of (a) was calculated to be $0.51 \%$, while (b), (c) and (d) were found to individually reduce the mismatch by $0.18 \%, 0.15 \%$ and $0.27 \%$, respectively. The extracted overall lattice mismatch of $-0.51 \%$ for the method (a) is in good agreement with the measured value from the sample using only the method (a) discussed before. It was found that the method (d) is the most effective in strain compensation as it provides In-Sb bonds at both sides of the inserted In layer. The method (b) is slightly more effective than the method (c). PL measurements were carried out and show peak wavelengths around 4.5

Table 2. Expressions of overall lattice mismatch in combined strain compensation schemes.

\begin{tabular}{|c|c|}
\hline Schemes & Overall lattice mismatch \\
\hline (b) $+(\mathrm{c})$ & $\begin{array}{l}f=\left[(M+x) f_{\text {InAs }}+\left(N z^{\prime}\right) f_{\text {GaAs }}+(2-x) f_{\text {InSb }}\right] /(M+N+2) \\
f=\left(0.1258-0.0062 M-0.0691 x-0.0726 N z^{\prime}\right) /(M+N+2)\end{array}$ \\
\hline (b) $+(d)$ & $\begin{array}{l}f=\left[\left(M-1+x+\frac{y}{2}\right) f_{\text {InAs }}+\left(\frac{y}{2}+N z^{\prime}\right) f_{\text {GAAs }}+\left(3-x-\frac{y}{2}\right) f_{\text {InSb }}\right] /(M+N+2) \\
f=\left(0.1949-0.0062 M-0.0691 x-0.0709 y-0.0726 N z^{\prime}\right) /(M+N+2)\end{array}$ \\
\hline$(c)+(d)$ & $\begin{array}{l}f=\left[\left(M+\frac{x}{2}\right) f_{\text {InAs }}+\left(\frac{x}{2}+N z^{\prime}\right) f_{\text {GaAs }}+\left(2-\frac{X}{2}\right) f_{\text {InSb }}\right] /(M+N+2) \\
f=\left(0.1258-0.0062 M-0.0709 x-0.0726 N z^{\prime}\right) /(M+N+2)\end{array}$ \\
\hline$(b)+(c)+(d)$ & $\begin{array}{l}f=\left[(M-1+x) f_{\text {InAs }}+\left(N z^{\prime}\right) f_{\text {GaAs }}+(3-x) f_{\text {InSS }}\right] /(M+N+3) \\
f=\left(0.1887-0.0062 M-0.0691 x-0.0726 N z^{\prime}\right) /(M+N+3)\end{array}$ \\
\hline
\end{tabular}


$\mu \mathrm{m}$ for samples grown with a combination of two methods, and higher intensity than that of the samples which were grown with only one method. To summarize, strain compensation strategies with a combination of inserting one monolayer $\mathrm{InSb}$ at one of the interfaces and one monolayer $\mathrm{InSb}$ in the $\mathrm{GaSb}$ layer are effective for both strain compensation and improvement of interface quality. In this way, the residual strain can be controlled between tensile and compressive type.

\section{Band Engineering of the Proposed T2SL Structures}

The insertion of $1 \mathrm{ML} \mathrm{InSb}$ in the GaSb layer of a T2SL structure has been found to be beneficial for both strain compensation and improving interface quality. The band structure of such T2SL structures has been calculated with a $\mathrm{k} \cdot \mathrm{p}$ model. Figures 8(a) and (b) show the alignments of the valence and conduction bands, as well as the envelope wavefunctions of a T2SL structure (10 ML InAs/5 ML GaSb/1 ML InSb/5 ML GaSb). It is found that the lowest lying conduction band state in the superlattice, namely $\mathrm{C} 1$, is a mixture of mainly bulk conduction band (C) and light hole ( $\mathrm{LH})$ states $(78 \%$ and $19 \%$, respectively), and very little of spin-orbit (SO) states. The lowest superlattice valence band state, $\mathrm{HH} 1$, on the

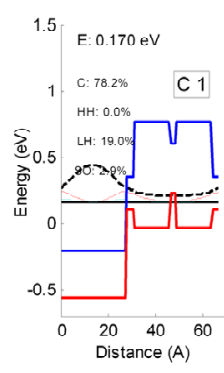

(a)

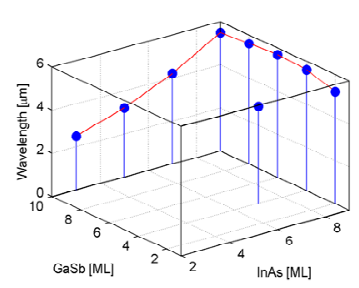

(d)

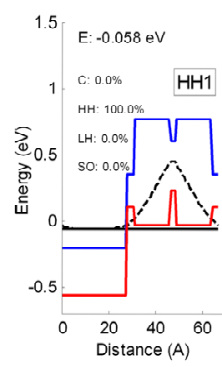

(b)

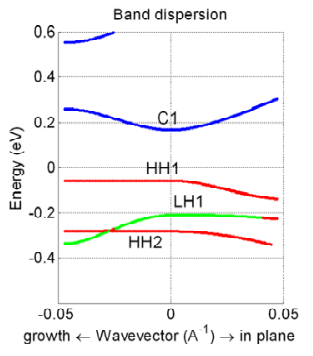

(c)

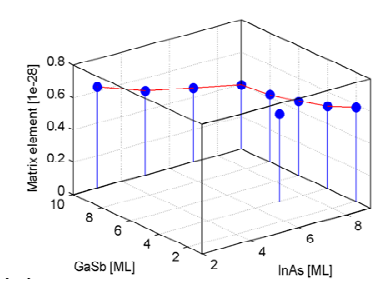

(e)

Figure 8. Band structure calculations based on a $\mathbf{k} \cdot \mathbf{p}$ model. (a) and (b) show band alignments of a T2SL structure with $10 \mathrm{ML}$ InAs/5 ML GaSb/1ML InSb/5 ML GaSb and the calculated energy levels and zone center envelope wavefunctions for the lowest confined levels in the conduction and the valence band, respectively. (c) shows the band dispersion of the same T2SL structure along, and perpendicular to, the growth direction. (d) and (e) show the cutoff wavelength and zone center optical matrix element $\|<\mathrm{C} 1|\boldsymbol{P}(\mathrm{k}=0)| \mathrm{HH} 1>\|$, respectively, as a function of InAs and GaSb thicknesses. other hand, is pure $100 \%$ heavy-hole $(\mathrm{HH})$ in character. The electron wavefunction extends into the $\mathrm{GaSb}$ layer, while that of holes in the valence band is confined mostly in the GaSb layer. The corresponding band structure is shown in Figure 8(c). The HH1 band is found to have very little dispersion in the growth direction, which means that the effective mass is large and that it is difficult for the holes to tunnel through the layers. InAs and GaSb thicknesses were varied individually in our calculations. Figure 8(d) shows the effective cutoff wavelength of the material as a function of layer thickness. Just as for T2SL structures without an InSb layer in the middle of GaSb layer, the superlattice bandgap is found to be sensitive only to the changes in the InAs thickness. The reason is that, due to the large heavy-hole effective mass of the GaSb valence band, an increased $\mathrm{GaSb}$ thickness leads only to a small upward shift of the HH1 energy. With increasing hole confinement layer (GaSb) thickness, the barrier strength for electrons increases, leading to reduced conduction band dispersion in the growth direction. The so created upward movement of the $\mathrm{C} 1$ band minimum just happens to almost perfectly match that of the HH1 energy.

The optical matrix element $\|<\mathrm{C} 1|P(\boldsymbol{k})| \mathrm{HH} 1>\|$ is an indication of optical transition strength. It can be found in Figure 8(e) that the strength of optical transition is indeed larger with thinner GaSb and InAs layers. The bandgap and optical transition can thus be optimized individually.

\section{Single Pixel Photodetector}

Single pixel $p-i-n$ photodetectors are demonstrated. The structure consists of 560 periods of $4 \mathrm{ML}$ InAs/8 ML GaSb T2SLs with strain compensation strategy of (b) + (c) $+(d)$. The process uses a combination of dry- and wetetching to create pixels. The mesa sidewalls are then passivated with polymer-based passivation and the contacts

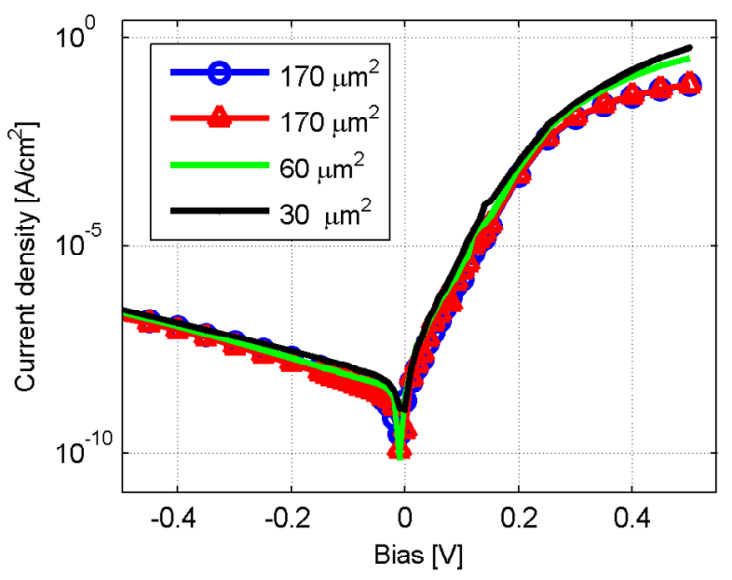

Figure 9. Measured dark current density for different pixel sizes at $80 \mathrm{~K}$. 
are deposited. The optical response is measured with a $100 \%$ cut off at $3.8 \mu \mathrm{m}$. Figure 9 shows the dark current density for different pixel sizes at $80 \mathrm{~K}$. The dark current density at small reverse biases is in the $10^{-9} \mathrm{~A} / \mathrm{cm}^{2}$ range at $80 \mathrm{~K}$ and increases to the $10^{-4} \mathrm{~A} / \mathrm{cm}^{2}$ range at $160 \mathrm{~K}$, and is independent of pixel size as a result of effective device passivation.

\section{Summaries}

In summary, we have investigated MBE growth conditions of InAs/GaSb T2SL materials aiming for MWIR FPA detectors in an As-dominated growth environment. The growth temperature and the soaking time were optimized in terms of interface, surface and transport quality. The optimal growth temperature was found at around $340^{\circ} \mathrm{C}-360^{\circ} \mathrm{C}$. The 12 -second $\mathrm{Sb}$ soaking on InAs was found to replace only $5 \%$ As atoms while the 0.5 second As soaking could replace $\mathrm{Sb}$ atoms up to $26 \%$. Different strain compensation strategies by inserting one monolayer of InSb at different positions and their combinations were proposed and tested to be effective. Combinations of the strain compensation methods were found to not only further compensate the tensile strain but also lead to high interface quality and PL intensity. The overall strain can be tuned from tensile type to compressive type without degradation of the interface quality. The effect of the strain compensation is well modeled by analytic functions and the average As compositions at interfaces or in $\mathrm{GaSb}$ can be extracted. Optical properties of the samples were examined by PL. The absorption wavelength can be controlled within the MWIR range by adjusting InAs and GaSb thicknesses. Calculations of the band structures indicate that the absorption wavelength depends primarily on the InAs thickness when inserting 1 ML InSb in the middle of the GaSb layer. Single pixel photodetectors with a low dark current were demonstrated.

The Swedish Governmental Agency for Innovation Systems (VINNOVA) is acknowledged for financial support of this project.

\section{Acknowledgements}

The Swedish Governmental Agency for Innovation Systems (VINNOVA) is acknowledged for financial support of this project.

\section{REFERENCES}

[1] G. Hasnain, B. Levine, D. Sivco, A. Cho, "Mid-Infrared Detectors in the 3 - $5 \mu \mathrm{m}$ Band Using Bound to Continuum State Absorption in InGaAs/InAlAs Multiquantum Well Structures," Applied Physics Letters, Vol. 56, No. 8, 1990, pp. 770-772. doi:10.1063/1.103186
[2] A. Rogalski, "Recent Progress in Infrared Detector Technologies," Infrared Physics \& Technology, Vol. 54, 2011, pp. 136-154. doi:10.1016/j.infrared.2010.12.003

[3] M. Z. Tidrow, W. A. Beck, W. W. Clark, H. K. Pollehn, J. W. Little, N. K. Dhar, R. P. Leavitt, S. W. Kennerly, D. W. Beekman, A. C. Goldberg and W. R. Dyer, "Device Physics and Focal Plane Array Applications of QWIP and MCT," Opto-Electronics Review, Vol. 7, No. 4, 1999, pp. 283-296.

[4] M. Walther, R. Rehm, J. Schmitz, J. Fleissner, F. Rutz, L. Kirste, R. Scheibner, J. Wendler and J. Ziegler,'Quantum Sensing and Nanophotonic Devices VII," SPIE, San Francisco, 2010.

[5] G. Sai-Halasz, R. Tsu and L. Esaki, "A New Semiconductor Superlattice," Applied Physics Letters, Vol. 30, No. 12, 1977, p. 651. doi:10.1063/1.89273

[6] D. Smith and C. Mailhiot, "Proposal for Strained Jour II Superlattice Infrared Detectors," Journal of Applied Physics, Vol. 62, No. 6, 1987, p. 2545. doi:10.1063/1.339468

[7] G. A. Sai-Halasz, L. Esaki and W. A. Harrison, "InAsGaSb Superlattice Energy Structure and Its Semiconductor-Semimetal Transition," Physical Review B, Vol. 18, No. 6, 1978, pp. 2812-2818. doi:10.1103/PhysRevB.18.2812

[8] Y. Wei, A. Gin, M. Razeghi and G. Brown, "Type II InAs/GaSb Superlattice Photovoltaic Detectors with Cutoff Wavelength Approaching $32 \mu \mathrm{m}$," Applied Physics Letters, Vol. 81, No. 19, 2002, p. 3675. doi:10.1063/1.1520699

[9] R. Rehm, M. Walther, J. Schmitz, J. Fleissner, J. Ziegler, W. Cabanski and R. Breiter, "Dual-Colour Thermal Imaging with InAs/GaSb Superlattices in Mid-Wavelength Infrared Spectral Range," Electronics Letters, Vol. 42, No. 10, 2006, pp. 577-578. doi:10.1049/el:20060878

[10] A. Khoshakhlagh, E. Plis, S. Myers, Y. D. Sharma, L. R. Dawson, S. Krishna, "Optimization of InAs/GaSb Type-II Superlattice Interfaces for Long-Wave $(\sim 8 \mu \mathrm{m})$ Infrared Detection" Journal of Crystal Growth, Vol. 311, No. 7, 2009, pp. 1901-1904. doi:10.1016/j.jcrysgro.2008.11.027

[11] A. Rogalski, J. Antoszewski, L. Faraone, "Third-Generation Infrared Photodetector Arrays," Journal of Applied Physics, Vol. 105, 2009, Article ID: 91101. doi:10.1063/1.3099572

[12] M. Walther, J. Schmitz, R. Rehm, S. Kopta, F. Fuchs, J. Fleissner, W. Cabanski and J. Ziegler, "Growth of InAs/ $\mathrm{GaSb}$ Short-Period Superlattices for High-Resolution Mid-Wavelength Infrared Focal Plane Array Detectors," Journal of Crystal Growth, Vol. 278, 2005, pp. 156-161. doi:10.1016/j.jcrysgro.2004.12.044

[13] M. Walther, R. Rehm, F. Fuchs, J. Schmitz, J. Fleißner, W. Cabanski, D. Eich, M. Finck, W. Rode, J. Wendler, R. Wollrab and J. Ziegler, "256 × 256 Focal Plane Array Midwavelength Infrared Camera Based on InAs/GaSb Short-Period Superlattices," Journal of Electronic Materials, Vol. 34, No. 6, 2005, pp. 722-725. doi:10.1007/s11664-005-0010-Z

[14] S. A. Pour, E. K. Huang, G. Chen, A. Haddadi, B. M. Nguyen and M. Razeghi, "High Operating Temperature 
Midwave Infrared Photodiodes and Focal Plane Arrays Based on Type-II InAs/GaSb Superlattices," Applied Physics Letters, Vol. 98, No. 14, 2011, pp. 143501143503. doi: $10.1063 / 1.3573867$

[15] R. Rehm, M. Walther, F. Rutz, J. Schmitz, A. Wörl, J. M. Masur, R. Scheibner, J. Wendler and J. Ziegler, "256 $\times$ 256 Focal Plane Array Midwavelength Infrared Camera Based on InAs/GaSb Short-Period Superlattices," Journal of Electronic Materials, Vol. 40, No. 6, 2011, pp. 17381743. doi:10.1007/s11664-011-1674-1

[16] S. D. Gunapala, D. Z. Ting, C. J. Hill, J. Nguyen, A. Soibel, S. B. Rafol, S. A. Keo, J. M. Mumolo, M. C. Lee, J. K. Liu and B. Yang, "Demonstration of a $1024 \times 1024$ Pixel InAs-GaSb Superlattice Focal Plane Array," IEEE Photonics Technology Letters, Vol. 22, No. 24, 2010, pp. 1856-1858. doi:10.1109/LPT.2010.2089677

[17] E. K. W. Huang, P. Y. Delaunay, B. M. Nguyen, S. A. Pour and M. Razeghi, "Photovoltaic MWIR Type-II Superlattice Focal Plane Array on GaAs Substrate," IEEE Journal of Quantum Electronics, Vol. 46, No. 12, 2010, pp. 1704-1708. doi:10.1109/JQE.2010.2061218

[18] N. Herres, F. Fuchs, J. Schmitz, K. M. Pavlov, J. Wagner, J. D. Ralston, P. Koidl, C. Gadaleta and G. Scamarcio, "Effect of Interfacial Bonding on the Structural and Vibrational Properties of InAs/GaSb Superlattices," Physical Review B, Vol. 53, No. 23, 1996, pp. 15688-15705.

[19] J. H. Li, D. W. Stokes, J. C Wickett, O. Caha, K. E. Bassler and S. C. Moss, "Effect of Strain on the Growth of InAs/GaSb Superlattices: An X-Ray Diffraction Study," Journal of Applied Physics, Vol. 107, No. 12, 2010, Article ID: 123504. doi:10.1063/1.3429100

[20] E. Plis, S. Annamalai, K. T. Posani, S. Krishna, R. A. Rupani and S. Ghosh, "Midwave Infrared Type-II InAs/ GaSb Superlattice Detectors with Mixed Interfaces," Journal of Applied Physics, Vol. 100, No. 1, 2006, Article ID: 14510 . doi:10.1063/1.2214222

[21] J. B. Rodriguez, P. Christol, L. Cerutti, F. Chevrier and A. Joullié, "MBE Growth and Characterization of Type-II InAs/GaSb Superlattices for Mid-Infrared Detection," Journal of Crystal Growth, Vol. 274, No. 1-2, 2005, pp. 6-13. doi:10.1016/i.jcrysgro.2004.09.088

[22] H. J. Haugan, L. Grazulis, G. J. Brown, K. Mahalingam, D. H. Tomich, "Exploring Optimum Growth for High Quality InAs/GaSb Type-II Superlattices," Journal of Crystal Growth, Vol. 261, No. 4, 2004, pp. 471-478. doi:10.1016/j.jcrysgro.2003.09.045

[23] E. Plis, A. Khoshakhlagh, S. Myers, H. S. Kim, N. Gautam, Y. D. Sharma, S. Krishna, S. J. Lee and S. K. Noh, "Molecular Beam Epitaxy Growth and Characterization of Type-II InAs/GaSb Strained Layer Superlattices for Long-Wave Infrared Detection," Journal of Vacuum Science \& Technology B, Vol. 28, No. 3, 2010, pp. C3-C13.

[24] Y. Wei, J. Bae, A. Gin, A. Hood, M. Razeghi, G. Brown and M. Tidrow, "High Quality Type II InAs/GaSb Superlattices with Cutoff Wavelength $\sim 3.7 \mu \mathrm{m}$ Using Interface Engineering," Journal of Applied Physics, Vol. 94, No. 7, 2003, p. 4720. doi:10.1063/1.1606506

[25] B. Satpati, J. Rodriguez, A. Trampert, E. Tournie, A. Joullie and P. Christol, "Interface Analysis of InAs/GaSb Superlattice Grown by MBE," Journal of Crystal Growth, Vol. 301-302, 2007, pp. 889-892. doi:10.1016/i.jcrysgro.2006.11.284

[26] E. Luna, B. Satpati, J. B. Rodriguez, A. N. Baranov, E. Tournié and A. Trampert, "Interfacial Intermixing in InAs/GaSb Short-Period-Superlattices Grown by Molecular Beam Epitaxy," Applied Physics Letters, Vol. 96, No. 2, 2010, Article ID: 021904.

[27] R. Kaspi, J. Steinshnider, M. Weimer, C. Moeller and A. Ongstad, "As-Soak Control of the InAs-on-GaSb Interface," Journal of Crystal Growth, Vol. 225, No. 2-4, 2001, pp. 544-549. doi:10.1016/S0022-0248(01)00950-2

[28] D. Hoffman, B. Nguyen, P. Delaunay, A. Hood, M. Razeghi and J. Pellegrino, "Beryllium Compensation Doping of InAs/GaSb Infrared Superlattice Photodiodes," Applied Physics Letters, Vol. 91, No. 14, 2007, Article ID: 143507. doi:10.1063/1.2795086

[29] L. Bürkle, F. Fuchs, J. Schmitz and W. Pletschen, "Control of the Residual Doping of InAs/(GaIn)Sb Infrared Superlattices," Applied Physics Letters, Vol. 77, No. 11, 2000, p. 1659. doi:10.1063/1.1310167

[30] S. Bandara, P. Maloney, N. Baril, J. Pellegrino and M. Tidrow, "Doping Dependence of Minority Carrier Lifetime in Long-Wave Sb-Based Journal Article II Superlattice Infrared Detector Materials," Optical Engineering, Vol. 6, No. 50, 2011, Article ID: 61015.

[31] M. Walther, R. Rehm, J. Schmitz, J. Niemasz, F. Rutz, A. Worl, L. Kirste, R. Scheibner, J. Wendler and J. Ziegler, "Defect Density Reduction in InAs/GaSb Journal Article II Superlattice Focal Plane Array Infrared Detectors," Proceedings of SPIE, Vol. 7945, 2011, Article ID: 79451.

[32] B. M. Nguyen, G. Chen, M. A. Hoang and M. Razeghi, "Growth and Characterization of Long-Wavelength Infrared Type-II Superlattice Photodiodes on a 3-in GaSb Wafer," IEEE Journal of Quantum Electron, Vol. 47, No. 5, 2011, pp. 686-690. doi:10.1109/JQE.2010.2103049

[33] S. H. Huang, G. Balakrishnan, A. Khoshakhlagh, A. Jallipalli, L. R. Dawson and D. L. Huffaker, "Strain Relief by Periodic Misfit Arrays for Low Defect Density GaSb on GaAs," Applied Physics Letters, Vol. 88, No. 13, 2006, Article ID: 131911. doi:10.1063/1.2172742

[34] A. S. Bracker, M. J. Yang, B. R. Bennett, J. C. Culbertson and W. J. Moore, "Surface Reconstruction Phase Diagrams for InAs, AlSb, and GaSb," Journal of Crystal Growth, Vol. 220, No. 4, 2000, pp. 384-392. 